\title{
Alendronate, a double-edged sword acting in the mevalonate pathway
}

\author{
PAOLA MAURA TRICARICO ${ }^{1}$, MARTINA GIRARDELLI ${ }^{2}$, GIULIO KLEINER ${ }^{2}$, ALESSANDRA KNOWLES ${ }^{2}$, \\ ERICA VALENCIC $^{2}$, SERGIO CROVELLA ${ }^{1,2}$ and ANNALISA MARCUZZI ${ }^{2}$ \\ ${ }^{1}$ Department of Medicine, Surgery and Health Science, University of Trieste, Trieste I-34127; \\ ${ }^{2}$ Department of Advanced Diagnostic and Clinical Trials, Institute for Maternal and Child Health, \\ IRCCS ‘Burlo Garofolo’, Trieste I-34137, Italy
}

Received August 8, 2014; Accepted April 30, 2015

DOI: $10.3892 / \mathrm{mmr} .2015 .3957$

\begin{abstract}
Aminobisphosphonate aledronate is a compound commonly used clinically for the treatment of osteoporosis and other bone diseases, as a result of it preventing bone resorption. However, in previous years it has also been used to obtain cellular and animal models of a rare genetic disorder termed Mevalonate Kinase Deficiency (MKD). MKD is caused by mutations affecting the mevalonate kinase enzyme, in the cholesterol pathway and alendronate can be used to biochemically mimic the genetic defect as it inhibits farnesyl pyrophosphate synthase in the same pathway. Despite evidence in favor of the inhibition exerted on the mevalonate pathway, there is at least one clinical case of MKD in which alendronate improved not only skeletal and bone fractures, as expected, but also MKD clinical features. Based on this finding, the present study assessed the anti-inflammatory properties of this aminobisphosphonate in vitro. No anti-inflammatory effects of alendronate were observed in the in vitro experiments. Since MKD lacks specific treatments, these results may assist scientists and physicians in making the decision as to the most suitable choice of therapeutic compounds for this neglected disease.
\end{abstract}

\section{Introduction}

Alendronate (Ald), is a molecule belonging to the aminobisphosphonate family and is commonly used in the clinical treatment of osteoporosis and other bone disorders, including Paget's disease $(1,2)$. Ald acts by reducing the resorptive activity and inducing accelerated programmed cell death

Correspondence to: Dr Annalisa Marcuzzi, Department of Advanced Diagnostic and Clinical Trials, Institute for Maternal and Child Health, IRCCS 'Burlo Garofolo', Via dell'Istria 65/1, Trieste I-34137, Italy

E-mail: annalisa.marcuzzi@burlo.trieste.it

Key words: alendronate, mevalonate kinase deficiency, programmed cell death, cytokines of osteoclasts. Besides the well-known antibone resorption activity, the side effects of aminobisphosphonates have been investigated previously and contrasting findings report their pro-inflammatory effects, renal toxicity and adverse reaction in the upper gastrointestinal tract of patients and animal models, although with certain differences depending on the compound and the type of administration (3-6).

These effects are caused, at a molecular level, by the inhibition of the farnesyl pyrophosphate synthase enzyme in the mevalonate pathway. The decreased number of prenylated GTP-binding proteins anchored to the membrane of osteoclasts $(7,8)$ may induce apoptosis and therefore, reduce bone resorption.

Malfunctions in the post-translational modification known as prenylation and the overproduction of several pro-inflammatory cytokines, including IL-1 $\beta$, are also suggested to be at the basis of Mevalonate Kinase Deficiency (MKD) (9). MKD is an autosomal recessively-inherited disease (OMIM \#610377) caused by mutations in the $M V K$ gene (12q24.11), which encodes the enzyme, mevalonate kinase (MK) in the mevalonate pathway (Fig. 1) (10-13).

Our previous studies developed cellular and animal models of MKD obtained following administration of the aminobisphosphonate, Ald or lovastatin (Lova). These inhibit the mevalonate pathway and allow partial reproduction of the biochemical defect characterizing patients with MKD $(14,15)$.

However, Cantarini et al (16) described a case report in which Ald was administered to a patient with MKD to prevent skeletal and bone fractures and this treatment markedly rescued the inflammatory symptoms and led to a disease remission period of several months (16). The positive effects of Ald suggested that this may be used as a potential therapeutic drug for MKD and not exclusively for bone disorders (16). However, this raises the issue of the apparently opposite roles of Ald in MKD. This aminobisphosphonate appears to exhibit contrasting effects, while having been reported to improve the clinical features of one patient, it has been extensively used to inhibit the mevalonate pathway in vivo and in vitro $(14,15)$.

The intriguing observation that Ald antagonizes the pro-inflammatory effects of the inhibited mevalonate pathway, prompted the present study to re-assess the activity of this 
compound, using an MKD cellular model (murine Raw 264.7 monocyte-macrophage cell line) and monocytes isolated from two patients with MKD.

Additionally, to avoid compound-dependent results and dissipate any question or controversy of the findings obtained, the pathway was inhibited using two different compounds, Ald and Lova. Once the pathway was inhibited, the acute phase was mimicked by administering a pro-inflammatory stimulus, lipopolysaccharide (LPS), shortly followed by the therapeutic administration of Ald. The analysis of four pro-inflammatory cytokines, interleukin (IL)-1 $\alpha$, IL-1 $\beta$, IL-6 and tumor necrosis factor (TNF)- $\alpha$, and programmed cell death (PCD), was used to assess the potential anti-inflammatory effects of Ald on these cell models of MKD.

\section{Materials and methods}

Chemicals. Unless otherwise stated, the reagents were purchased from Sigma-Aldrich (Milan, Italy). LPS (E. coli serotype 055:B5; $1 \mathrm{mg} / \mathrm{ml}$ stock in $\left.\mathrm{H}_{2} \mathrm{O}\right)$, Ald $(30 \mathrm{mM})$ and Lova $(50 \mathrm{mM})$ were dissolved in saline solution (Diaco SpA, Trieste, Italy).

Cell culture. The raw 264.7 cells (murine monocyte/macrophage cell line; Sigma-Aldrich) were cultured at $2.5 \times 10^{5}$ cells $/ \mathrm{ml}$ in Dulbecco's modified Eagle's medium, supplemented with $10 \%$ fetal bovine serum (FBS; Euroclone Spa, Milan, Italy) and $100 \mu \mathrm{M}$ Ald or $20 \mu \mathrm{M}$ Lova for $20 \mathrm{~h}$ at $37^{\circ} \mathrm{C}$ in a $5 \% \mathrm{CO}_{2}$ incubator. Following incubation, $10 \mu \mathrm{g} / \mathrm{ml}$ LPS was added for an additional $24 \mathrm{~h}$. Where appropriate, Ald was added at three different concentrations $(25,50$ or $100 \mu \mathrm{M})$ together with LPS in order to analyze its potential anti-inflammatory properties.

Two patients with MKD were diagnosed during the first year of life and followed-up at the Institute for Maternal and Child Health, IRCCS 'Burlo Garofolo', Trieste, Italy. The MKD diagnosis was confirmed by genetic analysis (Table I). These two patients were recruited to the present study at the age of 24 and 13. They had no recurrent infections and were not in the acute phase of the disease at the time of enrollment. The present study was approved by the ethical and scientific review board of the Institute for Maternal and Child Health, IRCCS 'Burlo Garofolo' (no. 185/8; 19/08/2008). For a child to be eligible, informed consent had to be obtained from the parents or caregivers. Monocytes were isolated from the two patients with MKD by selection with monoclonal CD14 antibody (mouse IgG2a)-conjugated microbeads (Miltenyi Biotec, Bergisch Gladbach, Germany), performed with manual columns, according to the manufacturer's instructions. The cells were subsequently cultured at $2.5 \times 10^{5}$ cells $/ \mathrm{ml}$ in RPMI-1640 medium, containing 10\% FBS (Euroclone Spa) and $1 \mu \mathrm{g} / \mathrm{ml}$ LPS for $24 \mathrm{~h}$. As for the Raw 264.7 cells, $50 \mu \mathrm{M}$ Ald was added together with LPS, where appropriate

At the end of the incubation periods, the supernatant was collected for the cytokine assay and the cells were pelleted for the PCD assay.

PCD assay. The PCD of the Raw 264.7 cells and the patient isolated monocytes were monitored by flow cytometry using double staining with Annexin V-fluorescein isothiocyanate and propidium iodide (PI; Apoptosis Detection kit; Immunostep,
Table I. $M V K$ gene mutations.

\begin{tabular}{lc}
\hline Patient & Mutation \\
\hline 1 & S135L/V377I \\
2 & V377I/I268T \\
\hline
\end{tabular}

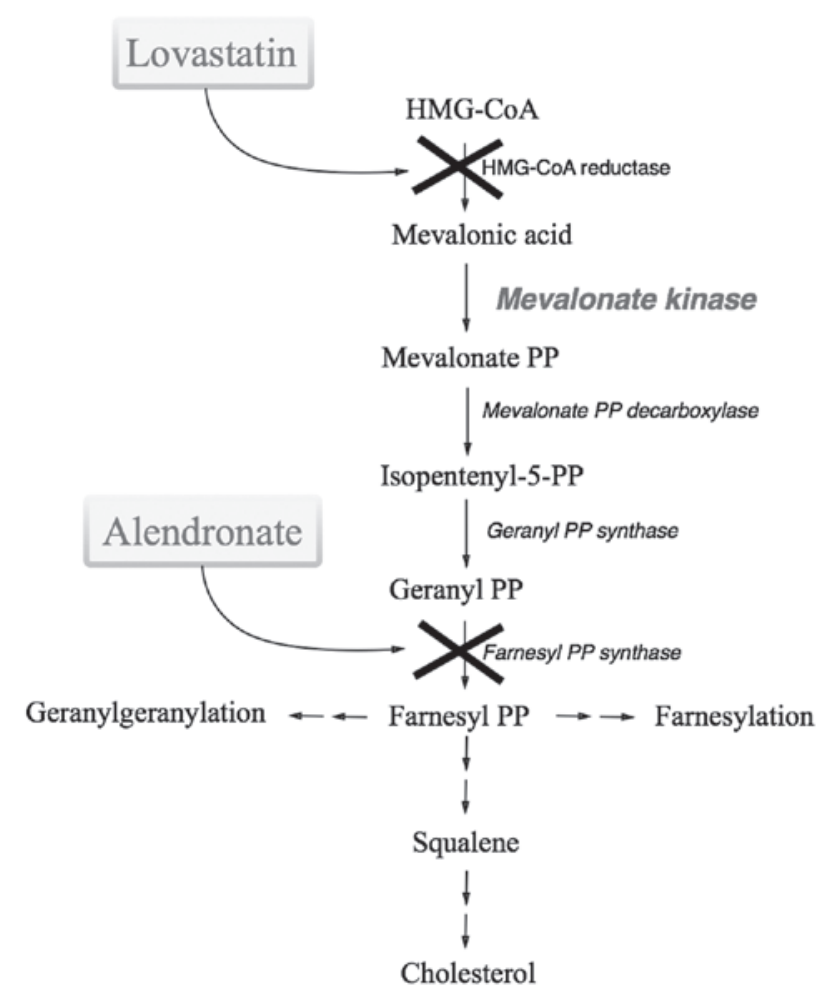

Figure 1. Schematic representation of the mevalonate pathway. The compounds used in the present study are indicated at the side of the pathway. Treatment with alendronate and lovastatin biochemically inhibited the pathway.

Salamanca, Spain), according to the manufacturer's instructions. The fluorescence was measured with a Cyan ADP cytometer and Summit version 4.3 software (Beckman Coulter, Fort Collins, CO, USA), and was subsequently analyzed with FlowJo 7.6 software (TreeStar Inc., Ashland, OR, USA). This technique was used to assess the effect of the treatments on cell viability. Debris were excluded from the plot based on the scatter (FSC vs. SSC) and the apoptotic (Annexin V positive, $\mathrm{A}^{+}$; PI negative, $\mathrm{PI}^{-}$and positive, $\left.\mathrm{PI}^{+}\right)$and the necrotic $\left(\mathrm{A}^{-}\right.$and $\mathrm{PI}^{+}$) cells were characterized based on the fluorescence emitted.

Cytokine production assay. The analysis of four pro-inflammatory cytokines, IL- $1 \alpha$, IL-1 $\beta$, IL- 6 and TNF- $\alpha$, was performed on culture medium from the Raw 264.7 cells and the patient isolated monocytes, using magnetic bead-based multiplex immunoassays (Bio-Plex; Bio-Rad Laboratories, Milano, Italy), according to the manufacturer's instructions. The data from the reactions were acquired using the Bio-Plex 200 reader, a digital processor managed the data output and Bio-Plex Manager 6.0 software (Bio-Rad) presented data as the Median Fluorescence Intensity and concentration (pg/ml). 
B
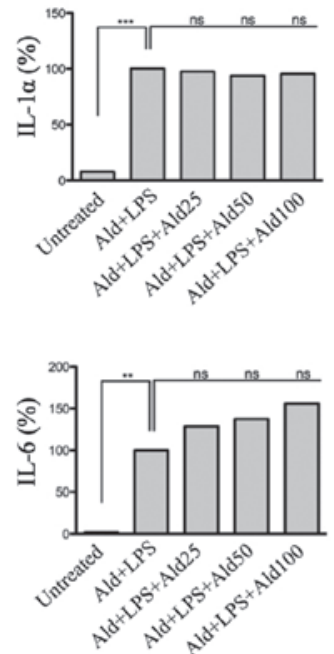

A
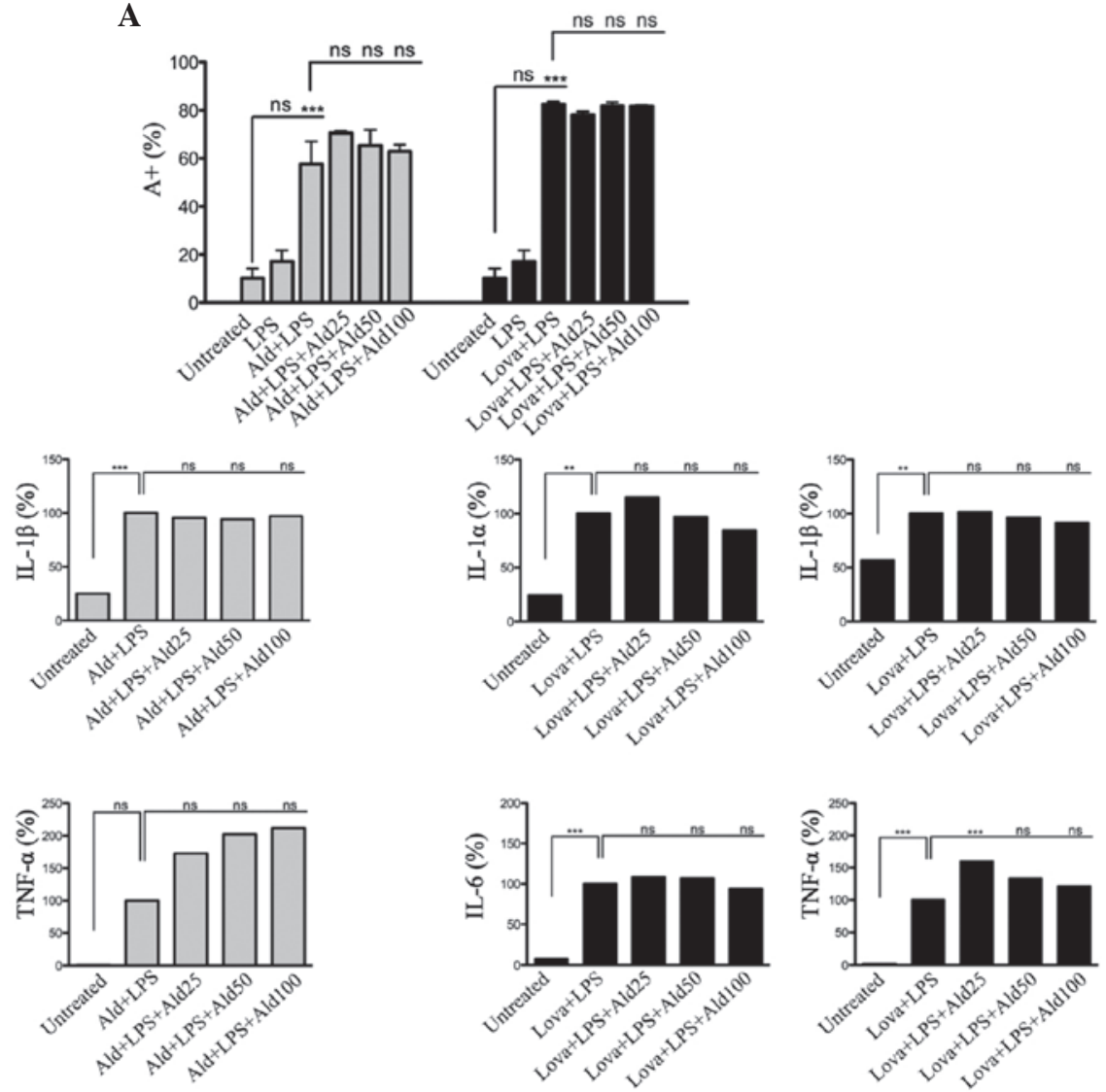

Figure 2. Raw 264.7 cells were incubated with $100 \mu \mathrm{M}$ Ald (left, grey) or $20 \mu \mathrm{M}$ Lova (right, black) and subsequently with $10 \mu \mathrm{g} / \mathrm{ml} \mathrm{LPS}$ and Ald $(25,50$ or $100 \mu \mathrm{M})$. (A) The percentage of apoptotic cells was detected by positive Annexin V staining $\left(\mathrm{A}^{+}\right)$and the data are expressed as the mean \pm standard deviation (B) The levels of IL-1 $\alpha$, IL-1 $\beta$, IL-6 and TNF- $\alpha$ in the supernatant was assessed. The data are demonstrated as the percentage of levels compared to the standard value (100\%). One-way analysis of variance and Bonferroni post-hoc test was performed for three independent experiments $\left({ }^{*} \mathrm{P}<0.05\right.$, ${ }^{* * *} \mathrm{P}<0.01$ and ${ }^{* * *} \mathrm{P}<0.001$, compared with the untreated group). Ald, alendronate; Lova, lovastatin; LPS, lipopolysaccharide; ns, not significant.

A

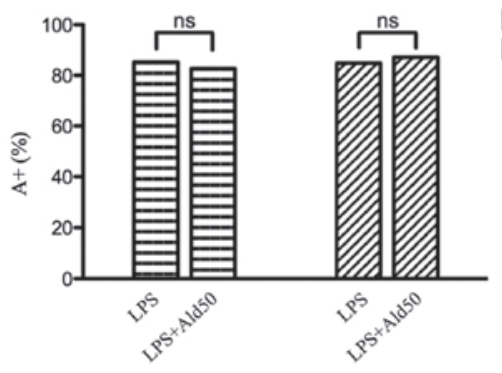

B

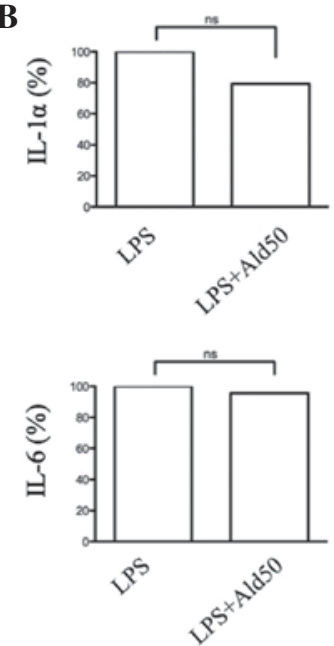

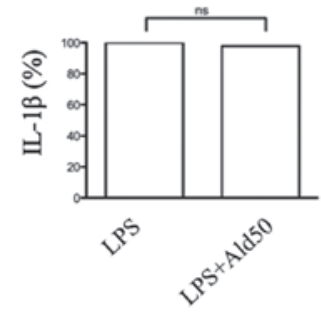

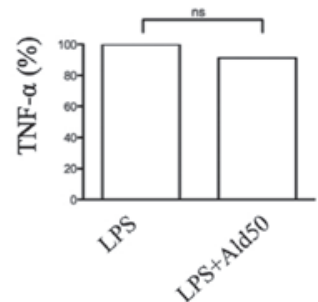

Figure 3. Monocytes from patients with Mevalonate Kinase Deficiency were incubated with $1 \mu \mathrm{g} / \mathrm{ml}$ LPS and $50 \mu \mathrm{M}$ Ald. (A) The percentage of apoptotic cells were determined by positive Annexin V ( $\left.\mathrm{A}^{+}\right)$staining. The data are expressed as the mean. (B) The levels of IL- $1 \alpha$, IL- $1 \beta$, IL- 6 and TNF- $\alpha$ in the supernatant were assessed. The data are demonstrated as the percentage of levels compared with LPS, considered as 100\% of cytokine production in the respective experimental setting. One-way analysis of variance and Bonferroni post hoc test was performed for three independent experiments. LPS, lipopolysaccharide; Ald, alendronate; ns, non significant.

Statistical analysis. The statistical significance was calculated using one-way analysis of variance and Bonferroni post-hoc test correction in the case of multiple comparisons, using GraphPad
Prism v5.0 software (GraphPad Software Inc., La Jolla, CA). The data are expressed as the mean \pm standard deviation. $\mathrm{P}<0.05$ was considered to indicate a statistically significant difference. 


\section{Results}

PCD. The Raw 264.7 cells demonstrated a statistically significant increase in PCD following the addition of a specific inhibitor (Ald or Lova) in addition to LPS. The Ald + LPS-treated or Lova + LPS-treated cells produced comparable results demonstrating that PCD was independent of the compound used to inhibit the pathway (Fig. 2A). Additionally, the production of the pro-inflammatory cytokines, IL-1 $\alpha$, IL-1 $\beta$, IL-6 and TNF- $\alpha$, was not normalized by the presence of Ald, regardless of which inhibitor was previously used (Fig. 2B).

Anti-inflammatory effect of Ald. In order to establish the potential anti-inflammatory effect of Ald, the drug was assessed at different concentrations $(25,50$ and $100 \mu \mathrm{M})$ in the murine cellular model. No affect was observed at any concentration with regards to the decrease of PCD or the secretion of pro-inflammatory cytokines (Fig. 2A and B).

Therapeutic activity of Ald. The effects of Ald on monocytes isolated from two different patients with MKD (Table I) were assessed. Following the addition of the pro-inflammatory stimulus, LPS, the potential therapeutic activity of Ald was assessed. As previously observed in the murine cell line, Ald revealed no improvement in the PCD in the monocytes from the patients with MKD, however, increased the percentage of apoptotic cells (Fig. 3A).

Cytokine levels. The production levels of the cytokines IL- $1 \alpha$, IL- $1 \beta$, IL- 6 and TNF- $\alpha$ were determined in the supernatant of the monocytes from the patients. The sensitivity of the specific human kit and their ranges were as follows: IL-1 $\alpha$ (1.4-22569 pg/ml), IL-1 $\beta$ (3.2-3261 pg/ml), IL-6 $(2.3-28880 \mathrm{pg} / \mathrm{ml})$ and TNF- $\alpha(5.8-95484 \mathrm{pg} / \mathrm{ml})$. The results were in the range of the limits of quantification and therefore, the data obtained demonstrated the inefficacy of Ald treatments in decreasing inflammation $(\mathrm{P}>0.05)$ (Fig. 3B).

\section{Discussion}

Despite the positive result described by Cantarini et al (16) in their case study, the present study failed to reproduce the anti-inflammatory effects of Ald in vitro, using a murine cell line or monocytes isolated from two patients with MKD. By contrast, treatment with Ald continued to be associated with increased levels of PCD and the production of inflammatory cytokines, suggesting the lack of anti-inflammatory activity for this compound, at least in vitro. A possible explanation for these contrasting findings may reside in the different backgrounds of the MVK gene. Indeed, the patient described by Cantarini et al and the two patients in the present study carry different mutations, the first being homozygous for V377I (16) and the latter being compound heterozygous (S135L/V377I and I268T/V377I).

In addition, patients with MKD exhibit a heterogeneous clinical phenotype, characterized by recurrent episodes of fever, irritability, lymphadenopathy, abdominal pain, diarrhoea and skin rash, which differs in terms of intensity and frequency from one patient to the other. Additionally, patients with MKD also exhibit marked variability in the response to therapies (statins and biological drugs) designed to rescue the inflammatory phenotype (17-19). According to Hoffmann et al (20), it is inappropriate to administer statins and/or aminobisphosphonate to patients with MKD exhibiting a genetically determined inhibited mevalonate pathway. However, the same author, reported discordant effects following the administration of statins. Indeed, certain patients with MKD exhibited an improvement of the clinical features, while other patients exhibited detrimental effects, including a marked increase of febrile attacks (20).

Despite isolated cases in which a variety of compounds have been demonstrated to improve the symptoms exhibited by patients, MKD still lacks standardized and targeted therapies and remains a neglected and disease, without a recommended therapeutic agent. Since our previous study reported an in vitro model useful to assess aminobisphosphonates, it was suggested to physicians to perform in vitro assays on monocytes isolated from patients with MKD. Therefore, a preliminary evaluation of the proper anti-inflammatory action of treatments may be obtained prior to treating patients themselves.

Being aware of all the limitations represented by cell models, in which the mevalonate pathway has been biochemically inhibited, the present study suggested that the in vitro model may contribute to identifying a common therapeutic strategy for patients with MKD.

\section{Acknowledgements}

This study was supported by a grant from the Institute for Maternal and Child Health, IRCCS 'Burlo Garofolo' (grant no. RC 42/11).

\section{References}

1. Maeda SS and Lazaretti-Castro M: An overview on the treatment of postmenopausal osteoporosis. Arq Bras Endocrinol Metabol 58: 162-171, 2014.

2. Silverman SL: Paget disease of bone: therapeutic options. J Clin Rheumatol 14: 299-305, 2008.

3. Sugawara S, Shibazaki M, Takada H, Kosugi H and Endo Y: Contrasting effects of an aminobisphosphonate, a potent inhibitor of bone resorption, on lipopolysaccharide-induced production of interleukin-1 and tumour necrosis factor alpha in mice. Br J Pharmacol 125: 735-740, 1998.

4. Amagase K, Inaba A, Senta T, et al: Gastric ulcerogenic and healing impairment effects of risedronate, a nitrogen-containing bisphosphonate in rats. Comparison with alendronate and minodronate. J Physiol Pharmacol 62: 609-618, 2011.

5. Richards PJ, Amos N, Williams AS and Williams BD: Pro-inflammatory effects of the aminobisphosphonate ibandronate in vitro and in vivo. Rheumatology (Oxford) 38: 984-991, 1999.

6. Diel IJ, Bergner R and Grötz KA: Adverse effects of bisphosphonates: current issues. J Support Oncol 5: 475-482, 2007.

7. Watts NB and Diab DL: Long-term use of bisphosphonates in osteoporosis. J Clin Endocrinol Metab 95: 1555-1565, 2010.

8. Fleisch H: Bisphosphonates: mechanisms of action. Endocr Rev 19: 80-100, 1998.

9. Frenkel J, Rijkers GT, Mandey SH, et al: Lack of isoprenoid products raises ex vivo interleukin-lbeta secretion in hyperimmunoglobulinemia D and periodic fever syndrome. Arthritis Rheum 46: 2794-2803, 2002.

10. Goldstein JL and Brown MS: Regulation of the mevalonate pathway. Nature 343: 425-430, 1990.

11. Drenth JP, van Deuren M, van der Ven-Jongekrijg J, Schalkwijk CG and van der Meer JW: Cytokine activation during attacks of the hyperimmunoglobulinemia $\mathrm{D}$ and periodic fever syndrome. Blood 85: 3586-3593, 1995. 
12. Haas D and Hoffmann GF: Mevalonate kinase deficiencies: from mevalonic aciduria to hyperimmunoglobulinemia D syndrome. Orphanet J Rare Dis 1: 13, 2006.

13. Mandey SH, Kuijk LM, Frenkel J and Waterham HR: A role for geranylgeranylation in interleukin-lbeta secretion. Arthritis Rheum 54: 3690-3695, 2006.

14. Marcuzzi A, Piscianz E, Kleiner G, et al: Clinical genetic testing of periodic Fever syndromes. Biomed Res Int 2013: 501305, 2013.

15. Tricarico PM, Marcuzzi A, Piscianz E, et al: Mevalonate kinase deficiency and neuroinflammation: balance between apoptosis and pyroptosis. Int J Mol Sci 14: 23274-23288, 2013.

16. Cantarini L, Vitale A, Magnotti F, et al: Weekly oral alendronate in mevalonate kinase deficiency. Orphanet J Rare Dis 8: 196, 2013.
17. Attout H, Guez S, Ranaivo I, Jameerbaccus N and Series C: A patient with hyper-IgD syndrome responding to simvastatin treatment. Eur J Intern Med 19: e82-e83, 2008.

18. Simon A, Drewe E, van der Meer JW, et al: Simvastatin treatment for inflammatory attacks of the hyperimmunoglobulinemia $D$ and periodic fever syndrome. Clin Pharmacol Ther 75: 476-483, 2004.

19. van der Hilst JC, Bodar EJ, Barron KS, et al: Long-term follow-up, clinical features and quality of life in a series of 103 patients with hyperimmunoglobulinemia D syndrome. Medicine (Baltimore) 87: 301-310, 2008

20. Hoffmann GF, Charpentier C, Mayatepek E, et al: Clinical and biochemical phenotype in 11 patients with mevalonic aciduria. Pediatrics 91: 915-921, 1993. 